POS $\quad$ PROCEEDINGS

\title{
Leptonic decays of $B_{(s)}$ and $D_{(s)}$ using the OK action
}

\author{
Sungwoo Park, Tanmoy Bhattacharya, Rajan Gupta \\ Theoretical Division T-2, Los Alamos National Laboratory, Los Alamos, NM 87545, USA \\ E-mail: sungwoo@lanl.gov, tanmoy@lanl.gov, rg@lanl.gov
}

\section{Yong-Chull Jang}

Physics Department, Brookhaven National Laboratory, Upton, NY 11973, USA

E-mail: ypj@bnl.gov

\author{
Benjamin J. Choi*, Seungyeob Jwa, Sunkyu Lee, Weonjong Lee \\ Lattice Gauge Theory Research Center, CTP, and FPRD, \\ Department of Physics and Astronomy, Seoul National University, Seoul 08826, South Korea \\ E-mail: wlee@snu.ac.kr \\ Jaehoon Leem \\ School of Physics, Korea Institute for Advanced Study (KIAS), Seoul 02455, South Korea \\ E-mail: leemjaehoon@kias.re.kr
}

\section{LANL-SWME Collaboration}

We present recent progress in the lattice calculation of leptonic decay constants for $B_{(s)}$ and $D_{(s)}$ mesons using the Oktay-Kronfeld $(\mathrm{OK})$ action for charm and bottom valence quarks, whose masses are tuned non-perturbatively. The calculations are done on 6 HISQ ensembles generated by the MILC collaboration with $N_{f}=2+1+1$ flavors. We also use the HISQ action for the light spectator quarks. Results are presented for the ratios $f_{B_{S}} / f_{B}$ and $f_{D_{s}} / f_{D}$, which reflect $S U$ (3) flavor symmetry breaking, and are independent of the renormalization constants of the axial currents.

37th International Symposium on Lattice Field Theory - Lattice2019

16-22 June 2019

Wuhan, China

${ }^{*}$ Speaker. 


\section{Introduction}

The decay constant $f_{P}$ of a pseudoscalar meson $P$ is defined by

$$
\left\langle 0\left|A^{\mu}\right| P\right\rangle=i p^{\mu} f_{P}
$$

where the external state $|P\rangle$ carries momentum $p^{\mu}$, and the axial current $A^{\mu}=\bar{\psi}_{h} \gamma^{\mu} \gamma_{5} \psi_{l}$. Here, the subscript $h(l)$ represents heavy (light) flavors in the $B_{(s)}$ and $D_{(s)}$ states. We use the OktayKronfeld (OK) action [1] for valence heavy quarks $\psi_{h}$ with $h=b, c$, and the HISQ action [2] for valence light quarks $\chi$ that are recast into the naive quark field $\psi_{l}$. The calculations are done on MILC HISQ ensembles with $N_{f}=2+1+1$ [3], whose parameters are summarized in Table 1 .

The OK action $S_{\mathrm{OK}}$ improves on the Fermilab formulation of the Wilson clover action [4] by including $\mathscr{O}\left(\lambda^{2}\right)$ and $\mathscr{O}\left(\lambda^{3}\right)$ improvement terms in heavy quark effective theory (HQET) power counting.

$$
\begin{array}{rlrl}
S_{\mathrm{OK}}=a^{4} \sum_{x} \bar{\psi}(x) & {\left[m_{0}+\gamma_{4} D_{4}\right.} & & \leftarrow \mathscr{O}\left(\boldsymbol{\lambda}^{0}\right) \\
& -\frac{1}{2} a \Delta_{4}+\zeta \boldsymbol{\gamma} \cdot \boldsymbol{D}-\frac{1}{2} r_{s} \zeta a \Delta^{(3)}-\frac{1}{2} c_{B} a \zeta i \boldsymbol{\Sigma} \cdot \boldsymbol{B} & & \leftarrow \mathscr{O}\left(\boldsymbol{\lambda}^{1}\right) \\
& -\frac{1}{2} c_{E} a \zeta \boldsymbol{\alpha} \cdot \boldsymbol{E} & & \leftarrow \mathscr{O}\left(\lambda^{2}\right) \\
& +c_{1} a^{2} \sum_{k} \gamma_{k} D_{k} \Delta_{k}+c_{2} a^{2}\left\{\boldsymbol{\gamma} \cdot \boldsymbol{D}, \Delta^{(3)}\right\}+c_{3} a^{2}\{\boldsymbol{\gamma} \cdot \boldsymbol{D}, i \boldsymbol{\Sigma} \cdot \boldsymbol{B}\} & \\
& \left.\left.+c_{E E} a^{2}\left\{\gamma_{4} D_{4}, \boldsymbol{\alpha} \cdot \boldsymbol{E}\right\}+c_{4} a^{3} \sum_{k} \Delta_{k}^{2}+c_{5} a^{3} \sum_{k} \sum_{j \neq k}\left\{i \Sigma_{k} B_{k}, \Delta_{j}\right\}\right] \psi(x)\right\} \cdot & \leftarrow \mathscr{O}\left(\boldsymbol{\lambda}^{3}\right)
\end{array}
$$

The definition of the operators in Eq. (1.2) can be found in Ref. [1]. The bare quark mass $m_{0}$ is related to the hopping parameter $\kappa$ as follows,

$$
a m_{0}=\frac{1}{2}\left(\frac{1}{\kappa}-\frac{1}{\kappa_{\text {crit }}}\right) .
$$

\begin{tabular}{l||l|l|l|lll}
\hline \hline ensemble ID & $a(\mathrm{fm})$ & $N_{s}^{3} \times N_{t}$ & $M_{\pi}(\mathrm{MeV})$ & $a m_{l}$ & $a m_{s}$ & $a m_{c}$ \\
\hline $\mathrm{a} 12 \mathrm{~m} 310$ & $0.1207(11)$ & $24^{3} \times 64$ & $305.3(4)$ & 0.0102 & 0.0509 & 0.635 \\
$\mathrm{a} 12 \mathrm{~m} 220$ & $0.1184(10)$ & $32^{3} \times 64$ & $216.9(2)$ & 0.00507 & 0.0507 & 0.628 \\
$\mathrm{a} 12 \mathrm{~m} 130$ & $0.1191(7)$ & $48^{3} \times 64$ & $131.7(1)$ & 0.00184 & 0.0507 & 0.628 \\
\hline $\mathrm{a} 09 \mathrm{~m} 310$ & $0.0888(8)$ & $32^{3} \times 96$ & $312.7(6)$ & 0.0074 & 0.037 & 0.440 \\
$\mathrm{a} 09 \mathrm{~m} 220$ & $0.0872(7)$ & $48^{3} \times 96$ & $220.3(2)$ & 0.00363 & 0.0363 & 0.430 \\
\hline $\mathrm{a} 06 \mathrm{~m} 310$ & $0.0871(6)$ & $48^{3} \times 144$ & $319.3(5)$ & 0.0048 & 0.024 & 0.286 \\
\hline \hline
\end{tabular}

Table 1: Parameters of the MILC HISQ ensembles with $N_{f}=2+1+1$ [3] used in our calculations. The lattice spacing $a$ is set by the Sommer scale $r_{1}$ and $N_{s}\left(N_{t}\right)$ is the lattice size in the spacial (temporal) direction. $M_{\pi}$ is the mass of Goldstone pions and $a m_{l}, a m_{s}$ and $a m_{c}$ are sea quark masses for the light (up and down), strange and charm quarks in lattice units, respectively. 
The non-perturbatively tuned hopping parameters for bottom and charm quarks, $\kappa_{b}, \kappa_{c}$, and the critical hopping parameter $\kappa_{\text {crit }}$ [5] for each measurement are summarized in Table 2.

In order to achieve a better overlap with the wave functions of the $B_{(s)}$ and $D_{(s)}$ meson states, we apply the covariant Gaussian smearing (CGS), $\left\{1+\sigma^{2} \nabla^{2} /\left(4 N_{\mathrm{GS}}\right)\right\}^{N_{\mathrm{GS}}}$ to the point source and sink as in Ref. [6]. The CGS parameters $\left\{\sigma, N_{\mathrm{GS}}\right\}$ for each measurement are given in Table 2. Here, we apply the CGS only to the heavy quark fields of the pseudoscalar interpolating operators.

\section{Correlator and current improvement}

The meson-meson (MM) and meson-current (MC) 2-point correlators are defined as follows [7],

$$
\begin{aligned}
C_{\mathrm{MM}}(t) & =\sum_{\mathbf{x}}\left\langle\mathscr{O}_{P}^{\dagger}(t, \mathbf{x}) \mathscr{O}_{P}(0)\right\rangle=\sum_{\alpha=1}^{4} \sum_{\mathbf{x}}\left\langle\mathscr{O}_{\alpha}^{\dagger}(t, \mathbf{x}) \mathscr{O}_{\alpha}(0)\right\rangle, \\
C_{\mathrm{MC}}(t)=\sum_{\mathbf{x}}\left\langle A^{4 \dagger}(t, \mathbf{x}) \mathscr{O}_{P}(0)\right\rangle & =\sum_{\alpha=1}^{4} \sum_{\mathbf{x}}\left\langle A_{\alpha}^{4 \dagger}(t, \mathbf{x}) \mathscr{O}_{\alpha}(0)\right\rangle,
\end{aligned}
$$

where the pseudoscalar heavy-light meson interpolating operator $\mathscr{O}_{\alpha}(t, \mathbf{x})$ and the axial current operator $A_{\alpha}^{4}(t, \mathbf{x})$ are

$$
\begin{aligned}
& \mathscr{O}_{\alpha}(t, \mathbf{x})=\left[\bar{\psi}(t, \mathbf{x}) \gamma_{5} \Omega(t, \mathbf{x})\right]_{\alpha} \chi(t, \mathbf{x}) \\
& A_{\alpha}^{4}(t, \mathbf{x})=\left[\bar{\Psi}(t, \mathbf{x}) \gamma^{4} \gamma_{5} \Omega(t, \mathbf{x})\right]_{\alpha} \chi(t, \mathbf{x})
\end{aligned}
$$

Here $\psi$ is the OK heavy quark field, $\chi$ is the HISQ light quark field, and

$$
\Omega(t, \mathbf{x}) \equiv \gamma_{1}^{x_{1}} \gamma_{2}^{x_{2}} \gamma_{3}^{x_{3}} \gamma_{4}^{t},
$$

and the subscript $\alpha$ represents the taste degree of the staggered light quarks. The rotated heavy quark field $\Psi$ is introduced to improve the axial current $A_{\alpha}^{4}$ up to $O\left(\lambda^{3}\right)$, the same level as the OK action.

$$
\begin{array}{rlrl}
\Psi(t, \mathbf{x})=( & 1 & \leftarrow O\left(\lambda^{0}\right) \\
& +d_{1} a \boldsymbol{\gamma} \cdot \boldsymbol{D} & & \leftarrow O\left(\lambda^{1}\right) \\
& +d_{2} a^{2} \Delta^{(3)}+d_{B} a^{2} i \boldsymbol{\Sigma} \cdot \boldsymbol{B}-d_{E} a^{2} \boldsymbol{\alpha} \cdot \boldsymbol{E} & & \leftarrow O\left(\lambda^{2}\right) \\
& +d_{r E} a^{3}\{\boldsymbol{\gamma} \cdot \boldsymbol{D}, \boldsymbol{\alpha} \cdot \boldsymbol{E}\}-d_{3} a^{3} \sum_{i} \gamma_{i} D_{i} \Delta_{i}-d_{4} a^{3}\left\{\boldsymbol{\gamma} \cdot \boldsymbol{D}, \Delta^{(3)}\right\} \\
& \left.-d_{5} a^{3}\{\boldsymbol{\gamma} \cdot \boldsymbol{D}, i \boldsymbol{\Sigma} \cdot \boldsymbol{B}\}+d_{E E} a^{3}\left\{\gamma_{4} D_{4}, \boldsymbol{\alpha} \cdot \boldsymbol{E}\right\}-d_{6} a^{3}\left[\gamma_{4} D_{4}, \Delta^{(3)}\right]\right\} & \\
& \left.-d_{7} a^{3}\left[\gamma_{4} D_{4}, i \boldsymbol{\Sigma} \cdot \boldsymbol{B}\right]\right) \psi(t, \mathbf{x}), & &
\end{array}
$$

where the improvement coefficients $d_{i}$ are given in Ref. [8]. 


\begin{tabular}{l|r|lll|l|r}
\hline \hline ensemble ID & $m_{x} / m_{s}$ & $\kappa_{\text {crit }}$ & $\kappa_{c}$ & $\kappa_{b}$ & $\left\{\sigma, N_{\mathrm{GS}}\right\}$ & $N_{\text {cfg }} \times N_{\text {src }}$ \\
\hline a12m310 & $1 / 5,1$ & 0.051211 & 0.048524 & 0.04102 & $\{1.5,5\}$ & $1053 \times 3$ \\
a12m220 & $1 / 10,1$ & 0.051218 & 0.048613 & 0.04070 & $\{1.5,5\}$ & $1000 \times 3$ \\
a12m130 & $1 / 27,1$ & 0.05119 & 0.048501 & 0.041343 & $\{1.5,5\}$ & $499 \times 3$ \\
\hline a09m310 & $1 / 5,1$ & 0.05075 & 0.04894 & 0.0429 & $\{2.0,10\}$ & $996 \times 3$ \\
a09m220 & $1 / 10,1$ & 0.05077 & 0.04902 & 0.0431 & $\{2.0,10\}$ & $1001 \times 3$ \\
\hline a06m310 & $1 / 5,1$ & 0.050357 & 0.04924 & 0.0452 & $\{3.0,22\}$ & $1017 \times 3$ \\
\hline \hline
\end{tabular}

Table 2: The $2^{\text {nd }}$ column gives the valence light quark masses $m_{x}$ and the following columns are the hopping parameters, CGS parameters and the number of measurements. $N_{\text {cfg }}$ represents the number of gauge configurations analyzed and $N_{\text {src }}$ is the number of sources used for measurement on each gauge configuration.

\section{Correlator fit}

We fit the 2-point correlation functions $C_{\mathrm{MM}}(t)$ and $C_{\mathrm{MC}}(t)$ with three even time-parity and two odd time-parity states and label it the $3+2$-state fit. The time parity is determined with respect to the shift operator in the Euclidean time direction. The fitting function is

$$
\begin{aligned}
& C_{\mathrm{Y}}(t)=g_{\mathrm{Y}}(t) \pm g_{\mathrm{Y}}(T-t), \quad(+ \text { for } \mathrm{MM},- \text { for } \mathrm{MC}) \\
& g_{\mathrm{Y}}(t)=A_{0}^{\mathrm{Y}} e^{-M_{0} t}[ 1+R_{2}^{\mathrm{Y}} e^{-\Delta M_{2} t}+R_{4}^{\mathrm{Y}} e^{-\left(\Delta M_{2}+\Delta M_{4}\right) t}+\cdots \\
&\left.-(-1)^{t} R_{1}^{\mathrm{Y}} e^{-\Delta M_{1} t}-(-1)^{t} R_{3}^{\mathrm{Y}} e^{-\left(\Delta M_{1}+\Delta M_{3}\right) t}+\cdots\right]
\end{aligned}
$$

where $\mathrm{Y}=\mathrm{MC}$ or $\mathrm{MM}, \Delta M_{i} \equiv M_{i}-M_{i-2}, M_{-1} \equiv M_{0}$, and

$$
A_{i}^{\mathrm{MM}} \equiv \frac{1}{2 M_{i}}\left\langle 0\left|\mathscr{O}_{P}\right| P_{i}\right\rangle\left\langle P_{i}\left|\mathscr{O}_{P}\right| 0\right\rangle, \quad A_{i}^{\mathrm{MC}} \equiv \frac{1}{2 M_{i}}\left\langle 0\left|A^{4}\right| P_{i}\right\rangle\left\langle P_{i}\left|\mathscr{O}_{P}\right| 0\right\rangle, \quad R_{i}^{\mathrm{Y}} \equiv \frac{A_{i}^{\mathrm{Y}}}{A_{0}^{\mathrm{Y}}}
$$

Here, $P_{i}$ represents the $i$-th excited meson state and $P_{0}$ the ground state. For a brevity, the subscript " 0 " for the ground state is dropped from now on. We take the following steps to analyze the 2-point correlation functions.

1. We fit the 2-point correlator, $C_{\mathrm{MM}}(t)$, data using the 3+2-state fit given in Eq. (3.1) to extract the ground state pseudoscalar meson mass $M\left(\equiv M_{0}\right)$ and amplitude $A^{\mathrm{MM}}\left(\equiv A_{0}^{\mathrm{MM}}\right)$ and control the excited states. We impose empirical Bayesian priors [9] on the excited state mass gaps $\Delta M_{i}$ and amplitude ratios $R_{i}^{\mathrm{MM}}$ to stabilize the fit. (See Fig. 1).

2. We feed the results for $M_{0}$, and $\Delta M_{i}$ obtained in the previous step as inputs into the fit for $C_{\mathrm{MC}}(t)$ to extract $A^{\mathrm{MC}}\left(\equiv A_{0}^{\mathrm{MC}}\right)$ and the ratios $R_{i}^{\mathrm{MC}}$. We use the same fit range and fit functional form as taken for $C_{\mathrm{MM}}(t)$

An example of the effective mass plot with

$$
m_{\mathrm{eff}}^{Y}(t) \equiv \frac{1}{2} \log \left[\frac{C_{Y}(t)}{C_{Y}(t+2)}\right]
$$

for the 2-point correlators $C_{\mathrm{MM}}(t)$ and $C_{\mathrm{MC}}(t)$ is shown in Fig. 2 along with the fits to them. 


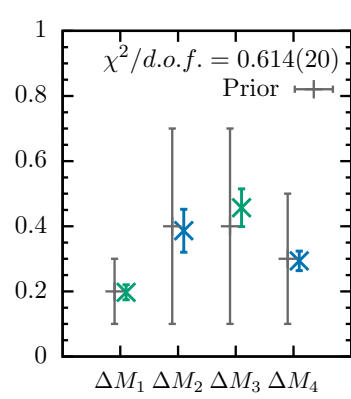

(a) $B$ meson

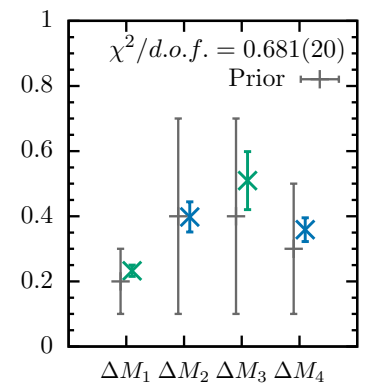

(b) $B_{s}$ meson

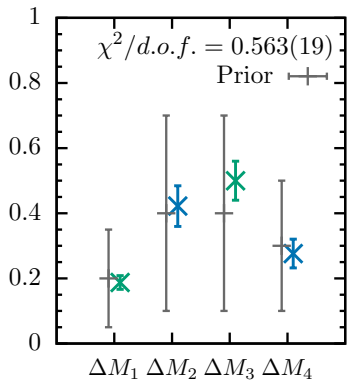

(c) $D$ meson

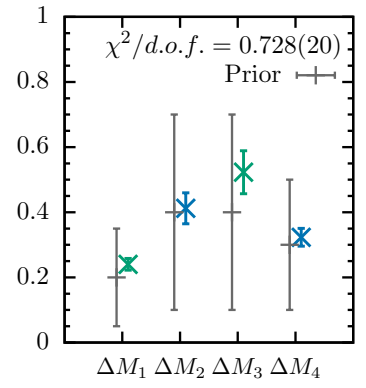

(d) $D_{s}$ meson

Figure 1: Fit results and Bayesian priors for $\Delta M_{i}$ from $a 12 m 130$ ensembles.
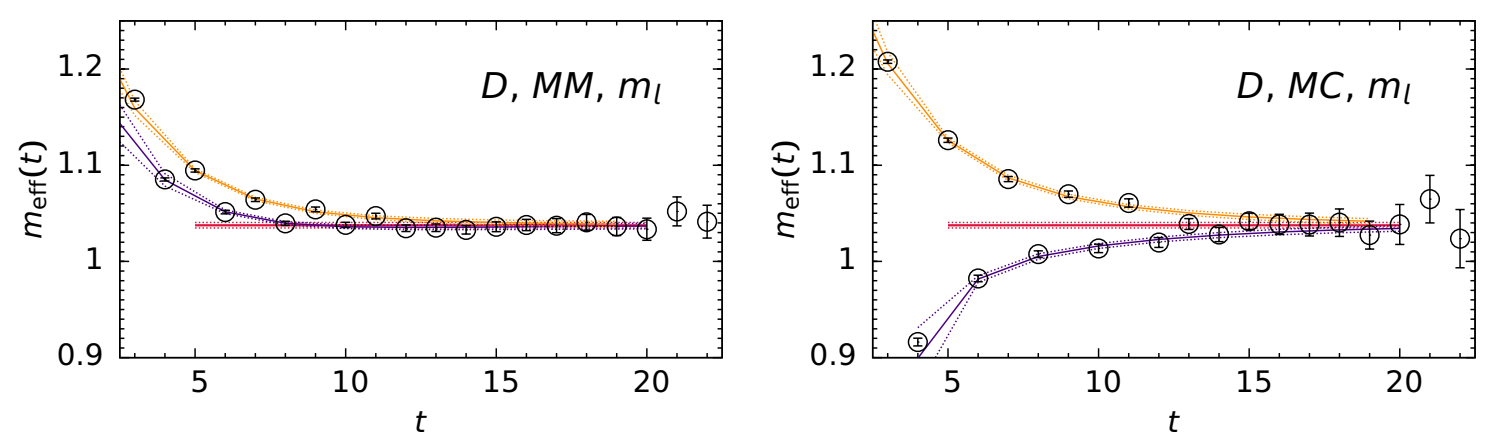

Figure 2: Effective mass plots for the $C_{\mathrm{MM}}(t)$ and $C_{\mathrm{MC}}(t)$ correlators of the $D$ meson on the $a 12 m 130$ ensemble with $m_{l}=m_{s} / 27$. The orange (purple) curves connect 3+2-state fit results on the odd (even) time slices. The horizontal red line shows the ground state mass $M_{0}$ within the fit range $\left[t_{\min }, t_{\max }\right]=[5,20]$. The axial current operators are improved up to the $\lambda^{3}$ order.

\section{Results}

The decay constant $f_{P}$ defined in Eq. (1.1) can be expressed in terms of the ground state amplitudes $A^{\mathrm{MM}}$ and $A^{\mathrm{MC}}$ as follows,

$$
f_{P}=Z_{A^{4}}^{h l} \sqrt{\frac{2}{M_{P}}} \frac{A^{\mathrm{MC}}}{\sqrt{A^{\mathrm{MM}}}},
$$

where we take the meson mass $M_{P}=M_{0}$ of the ground state obtained from the fits to $C_{\mathrm{MM}}$. The treelevel renormalization factor is given as $Z_{A^{4}}^{h l \text {,tree }}=e^{m_{1}^{h} / 2}$ where $m_{1}^{h}=\log \left(1+m_{0}^{h}\right)$ is the rest mass and $m_{0}^{h}$ is the bare mass for the heavy quark [4]. The perturbative and non-perturbative determination of $Z_{A^{4}}^{h l}$ is in progress. In this work, we present the flavor $S U(3)$ breaking ratio of decay constants:

$$
f_{X_{s}} / f_{X}=\frac{Z_{A^{4}}^{h s}}{Z_{A^{4}}^{h l}} \sqrt{\frac{M_{X}}{M_{X_{s}}}} \sqrt{\frac{A^{\mathrm{MM}}}{A_{s}^{\mathrm{MM}}}} \frac{A_{s}^{\mathrm{MC}}}{A^{\mathrm{MC}}} \cong \sqrt{\frac{M_{X}}{M_{X_{s}}}} \sqrt{\frac{A^{\mathrm{MM}}}{A_{s}^{\mathrm{MM}}}} \frac{A_{s}^{\mathrm{MC}}}{A^{\mathrm{MC}}},
$$

for $X_{(s)}=B_{(s)}$ and $D_{(s)}$ mesons. Here, $A_{s}^{\mathrm{MM}}$ and $A_{s}^{\mathrm{MC}}$ are the ground state amplitudes for the heavy-strange mesons. In this ratio, we assume that the light quark mass $m_{l}$ dependence of $Z_{A^{4}}^{h l}$ is negligible [7], so the ratio $Z_{A^{4}}^{h s} / Z_{A^{4}}^{h l} \cong 1$.

Fig. 3 shows that in the ratios $f_{B_{s}} / f_{B}$ and $f_{D_{s}} / f_{D}$, the effect of the current improvement applied to the heavy quark field (as given in Eq. (2.6)) cancels up to $O\left(\lambda^{3}\right)$. 


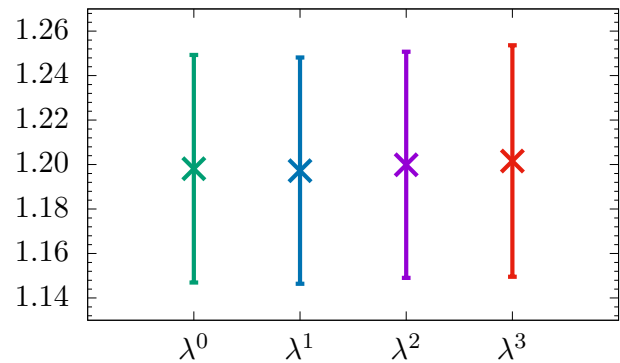

(a) $f_{B_{s}} / f_{B}$

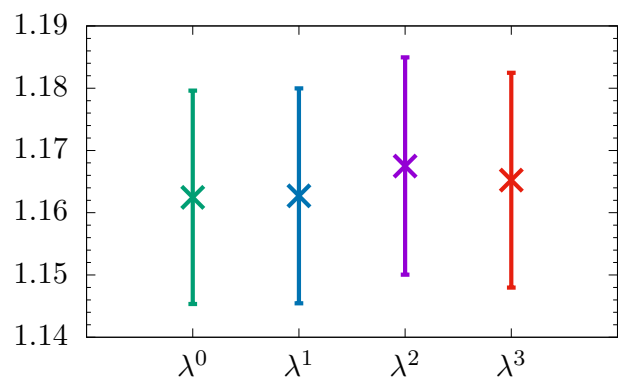

(b) $f_{D_{s}} / f_{D}$

Figure 3: The ratios of decay constants, $f_{B_{s}} / f_{B}$ and $f_{D_{s}} / f_{D}$, on the a $12 \mathrm{~m} 130$ ensemble as a function of current improvement order in HQET power counting.

In Fig. 4, we present preliminary results on $f_{B_{s}} / f_{B}$ and $f_{D_{s}} / f_{D}$ calculated on 6 different HISQ ensembles, and compare them with the continuum limit value given in the FLAG 2019 review [10]. The statistical errors in $f_{B_{s}} / f_{B}$ (Fig. 4 (a)) are much larger than in $f_{D_{s}} / f_{D}$ (Fig. 4 (b)). As a result, discerning a chiral or discretization effect in $f_{B_{S}} / f_{B}$ is not clear, other than to note that the result from the physical ensemble $a 12 \mathrm{~m} 130$ is consistent with the FLAG 2019 value. The results for $f_{D_{s}} / f_{D}$ show no significant discretization effect on the three lattices with $M_{\pi} \approx 310 \mathrm{MeV}$ and the two with $M_{\pi} \approx 220 \mathrm{MeV}$. On the other hand, there is a shift upwards towards the FLAG result as $M_{\pi}$ is lowered towards the physical value. Presuming that the OK action has significantly eliminated the heavy quark discretization error even on the coarsest lattice spacing $a \approx 0.12 \mathrm{fm}$ [11], the leading effect to quantify is the pion mass dependence. For the $a \approx 0.12 \mathrm{fm}$ data, the current trend is anchored by the physical ensemble with a value close to the FLAG 2019 result. In near future, we plan to add measurements on more ensembles to check for discretization effects and on more physical pion mass ensembles to improve the chiral-continuum extrapolation.

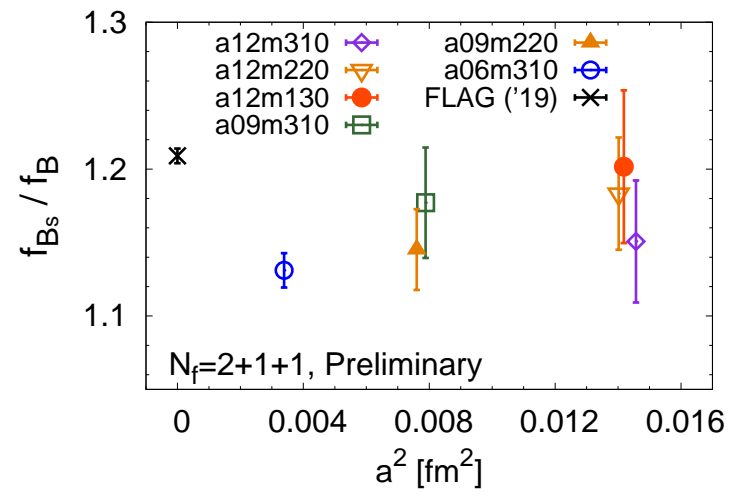

(a) $f_{B_{s}} / f_{B}$

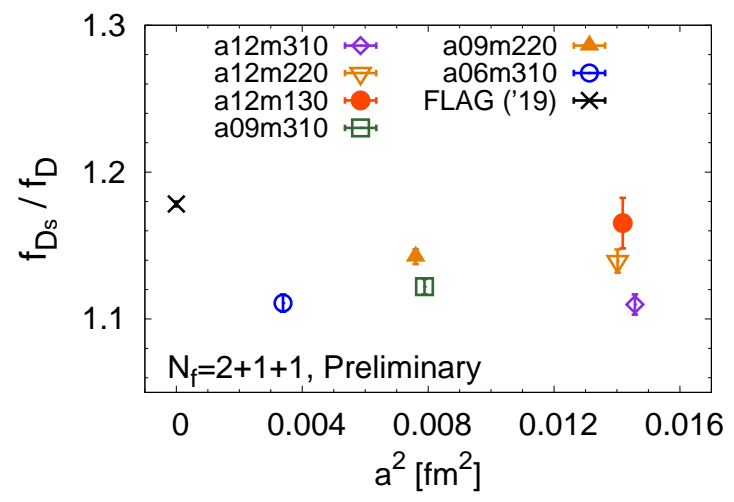

(b) $f_{D_{s}} / f_{D}$

Figure 4: The ratios of decay constants, $f_{B_{s}} / f_{B}$ and $f_{D_{s}} / f_{D}$ on six ensembles. The errors are purely statistical. The FLAG 2019 [10] results are given for the physical value. 


\section{Acknowledgments}

We thank the MILC collaboration for sharing the HISQ ensembles with us. Computations for this work were carried out in part on (i) facilities of the USQCD collaboration, which are funded by the Office of Science of the U.S. Department of Energy, (ii) the Nurion supercomputer at KISTI and (iii) the DAVID GPU clusters at Seoul National University. The research of W. Lee is supported by the Mid-Career Research Program (Grant No. NRF-2019R1A2C2085685) of the NRF grant funded by the Korean government (MOE). This work was supported by Seoul National University Research Grant in 2019. W. Lee would like to acknowledge the support from the KISTI supercomputing center through the strategic support program for the supercomputing application research (No. KSC-2017-G2-0009). T. Bhattacharya and R. Gupta were partly supported by the U.S. Department of Energy, Office of Science, Office of High Energy Physics under Contract No. DE-AC52-06NA25396. S. Park, T. Bhattacharya, R. Gupta and Y.-C. Jang were partly supported by the LANL LDRD program. Y.-C. Jang is partly supported by U.S. Department of Energy under Contract No. DE-SC0012704.

\section{References}

[1] M. B. Oktay and A. S. Kronfeld, New lattice action for heavy quarks, Phys. Rev. D78 (2008) 014504, [0803.0523].

[2] E. Follana, Q. Mason, C. Davies, K. Hornbostel, G. P. Lepage, J. Shigemitsu, H. Trottier, and $\mathrm{K}$. Wong, Highly improved staggered quarks on the lattice, with applications to charm physics, Phys. Rev. D75 (2007) 054502, [hep-lat/ 06100 92].

[3] A. Bazavov et al., Lattice QCD ensembles with four flavors of highly improved staggered quarks, Phys. Rev. D87 (2013), no. 5 054505, [1212.4768].

[4] A. X. El-Khadra, A. S. Kronfeld, and P. B. Mackenzie, Massive fermions in lattice gauge theory, Phys. Rev. D55 (1997) 3933-3957, [hep-lat/ 9604004 ].

[5] J. A. Bailey, T. Bhattacharya, R. Gupta, Y.-C. Jang, W. Lee, J. Leem, S. Park, and B. Yoon, Calculation of $\bar{B} \rightarrow D^{*} \ell \bar{v}$ form factor at zero recoil using the Oktay-Kronfeld action, EPJ Web Conf. 175 (2018) 13012, [1711.01786].

[6] B. Yoon et al., Controlling Excited-State Contamination in Nucleon Matrix Elements, Phys. Rev. D93 (2016), no. 11 114506, [1602.07737].

[7] A. Bazavov et al., B- and D-meson decay constants from three-flavor lattice QCD, Phys. Rev. D85 (2012) 114506, [1112.3051].

[8] J. A. Bailey, Y.-C. Jang, S. Lee, W. Lee, and J. Leem, Improvement of heavy-heavy and heavy-light currents with the Oktay-Kronfeld action, 2001.05590.

[9] B. Yoon et al., Isovector charges of the nucleon from 2+1-flavor QCD with clover fermions, Phys. Rev. D95 (2017), no. 7 074508, [1611.07452].

[10] S. Aoki et al., FLAG Review 2019, 1902.08191.

[11] J. A. Bailey, C. DeTar, Y.-C. Jang, A. S. Kronfeld, W. Lee, and M. B. Oktay, Heavy-quark meson spectrum tests of the Oktay-Kronfeld action, Eur. Phys. J. C77 (2017), no. 11 768, [1701.00345]. 